Objective There is conflicting evidence on the association between television viewing in childhood and cognition. No recent UK and few worldwide studies have looked at the longer-term effects of television viewing in childhood. This study investigates the association between verbal and non-verbal cognitive outcomes at 7 years and television habits at 3,5 and 7 years.

Method The study comprises longitudinal and cross-sectional analysis of the United Kingdom Millennium Cohort Study based on 8,148 children with complete data on variables of interest, maternal reports of television viewing and scores from objective tests of cognitive ability (British Ability Scales Second Edition) collected when cohort members were 3, 5 and 7 years. Mean ability scores were converted into the equivalent progress expected in a child at each age group using existing age equivalents derived for the cohort study population. Linear regression was used to estimate the relationship between each subscale and categories of television viewing (in relation to a reference group who watched between 1-3 hours of television a weekday) after adjustment for co-variates.

Results Children who did not watch television at 3 years had verbal ability scores at 7 years approximately 7 months behind the reference group ( $p<0.05$ ); their non-verbal ability skills were approximately 18 months delayed $(p<0.05)$. Children who watched less than 1 hour a weekday had delayed non-verbal skills of approximately 2 months ( $<<0.05)$

There was no significant association between television viewing at 5 and verbal or non-verbal ability at 7 years.

Children who did not watch television at 7 years had verbal ability scores approximately 3 months ahead of the reference group $(p<0.05)$. Those who watched less than 1 hour a weekday were approximately 1 month ahead $(p<0.05$ ). Children who watched over 3 hours of television a weekday had delays of more than 1 month $(p<0.05)$. There was no significant association between television viewing at 7years and non-verbal ability.

Conclusion There was a positive association between television viewing at 3 years and verbal and non-verbal cognition at 7 years in this cohort but a negative association between television viewing at 7 years and verbal skills at 7 years. This may influence potential guidance on television viewing. Possible explanations are the type of programmes watched or accumulation of viewing hours.

\section{G212 PAEDIATRIC ASSESSMENTS IN CHILD PROTECTION: HOW CAN WE MEASURE QUALITY?}

doi:10.1136/archdischild-2013-304107.224

1,2R Crowley, 1,2M Hyrapetian, 1,2E Day, 'D Hodes. 'Community Child Health, NHS Camden Provider Services, London, UK; ${ }^{2}$ School of Paediatrics, London Deanery, London, UK

Aims Child Protection is behind other paediatric specialties in the development of tools to measure the quality of services. We therefore aimed to develop quality indicators in Child Protection that could be used to evaluate paediatric assessments. This study shows how such tools were applied in two Child Protection services and how the resulting outcome data was used to guide service improvement.

Methods A menu of quality measures for paediatric assessment in child protection was formulated from the available guidance and evidence base. Indicators selected by the local team were incorporated into the existing paediatric assessment proforma used for children with suspected maltreatment. The indicators were chosen to be meaningful for the service and to include process as well as outcome measures, without being time-consuming for clinicians. Local teaching sessions informed all doctors about the project and administrators prospectively extracted data for collation in a central database.

Results 84 proformas completed between March and June 2012 were analysed using monthly run charts. Three quality indicators largely within the doctors' control - whether the child was offered a chance to speak to the doctor alone, choose who was present during examination, and attend with a parent or carer - showed some trend towards improvement, which might be expected as a consequence of the 'checklist' function of the proforma. By contrast, the proportion of children whose strategy meeting was attended by a doctor - which requires robust interagency communication in addition to individual action - showed no improvement. Freetext boxes gave qualitative information that has stimulated service improvement, such as ensuring access to a camera to record suspected physical maltreatment. Evidence of ambiguity in one question and suboptimal rates of questionnaire completion have led to modifications in the current cycle of data collection in 2013, the results of which will also be presented and compared.

Conclusions The quality of paediatric assessments can be measured through a clinician-led initiative. We demonstrated that measurement directly stimulates change, particularly when checklists are employed, and can identify specific areas for service improvement. This strategy could be used in other regions and in other areas of child protection.

\section{G213 HOW DOES THE PATTERN OF BRUISING RELATE TO THE MECHANISM OF INJURY IN CHILDREN?}

doi:10.1136/archdischild-2013-304107.225

RE Watson, S Maguire, A Kemp. Institute of Primary Care and Public Health, Cardiff University, Cardiff, UK

Objective It is essential that paediatricians assessing a child with bruising in suspected physical abuse can identify when the explanation for the bruise is implausible. Several published studies describe bruising patterns in populations of abused and non-abused children but we are not aware of any that describe the relationship between mechanism of injury and bruise pattern.

Methods Details of the mechanism of unintentional injury, the number of bruises, location and size were recorded for: children aged 0-11 years attending a Children's Emergency Department (ED) at a tertiary centre and children in the community (4-11 years at school, 0-4 year olds at two nurseries) with bruises of known cause. Students T-test, Chi-squared and Fisher's exact test were used to analyse associations between mechanisms and bruise patterns.

Results 351 children were recruited. Nine mechanisms of injury were recorded (Table 1). 81\% ( $p<0.001$ ) of non-ambulant children (mean age $=9.9$ months, $\mathrm{SD}=6.3$ ) sustained impact injuries whilst $60 \%$ of ambulant children (mean age $=41.7$ months, $\mathrm{SD}=30.4$ ) fell from $<1 \mathrm{~m}$ or standing height $(\mathrm{p}<0.001)$. The majority of children presented with small (mean size $=17.4 \mathrm{~mm}$, range $=2-115 \mathrm{~mm}$ (ED), $1-75 \mathrm{~mm}$ (Community)), and single (86.9\%) bruises related to each incident.

Falls from $<1 \mathrm{~m}$, falls from standing height hitting an object and impact injuries accounted for $79.5 \%$ (279/351) of all incidents. Bruises were most commonly found on the forehead ( $\mathrm{n}=114$, $29.7 \%$ ) knees and shins ( $\mathrm{n}=88,22.9 \%$ ) and head $(\mathrm{n}=39,10.2 \%)$ whilst bruising to certain areas were rare e.g. the front trunk (1.3\%), back of thigh $(0.9 \%)$ and genitalia $(0.3 \%)$.

Conclusion The data demonstrate predominant injury mechanisms for unintentional injury in children that relate to child's level of development. Multiple bruises from a single incident were rare, bruises were small and predominantly to the forehead, knees and shins and head. This predictable pattern of unintentional bruising can aid clinicians to judge the likelihood that a pattern of bruising is consistent with the mechanism of injury, and improve the detection of physical abuse where bruising pattern does NOT fit with a proposed mechanism. 